\title{
La didactique : essentielle, mais menacée
}

\author{
Marcel Thouin \\ Université de Montréal, Québec, Canada
}

\section{Pour citer cet article :}

Thouin, M. (2020). La didactique : essentielle, mais menacée. Didactique, 1(1), 61-86. doi : $10.37571 / 2020.0104$

Résumé : Les connaissances dont les êtres humains ont eu besoin pendant la plus grande partie de leur existence sur Terre sont très différentes des savoirs de la communauté scientifique du $21^{\mathrm{e}}$ siècle. La didactique a développé plusieurs théories et concepts, tels que le contrat didactique, la dévolution, la transposition didactique, les situations-problèmes et les modèles de changement conceptuel, qui visent à favoriser le passage de ces connaissances primordiales (dans le sens premier de « qui est le plus ancien ») vers les savoirs contemporains. Toutefois, malgré son rôle essentiel, tant dans le milieu scolaire que dans la formation des enseignants, la didactique est menacée. Une revalorisation de la didactique serait donc souhaitable.

Mots-clés : Connaissances primordiales; Théories et concepts de la didactique;

Didactique menacée 


\section{Introduction}

Selon Guy Brousseau (1983), qui peut être considéré comme l'un de ses fondateurs, l'objet principal de la didactique est d'étudier les situations ou les problèmes proposés à l'élève pour favoriser l'apparition, le fonctionnement et le rejet de ses conceptions successives (qui sont souvent erronées). En d'autres termes, la didactique s'intéresse principalement à l'évolution graduelle des conceptions des élèves, qu'on appelle souvent aussi le changement conceptuel.

Cette vision de la didactique s'avère des plus pertinentes quand on l'examine selon une perspective évolutionniste. En effet, cette perspective fait ressortir les nombreuses dissonances entre les facultés cognitives dont les êtres humains ont eu besoin pendant la plus grande partie de leur existence sur Terre et celles qui leur sont nécessaires de nos jours. Elle explique à quel point l'enseignement et l'apprentissage des disciplines scolaires contemporaines représentent un immense défi, tant pour les enseignants que pour les élèves.

Cet article, basé sur une analyse de contenu, vise d'abord à donner un aperçu des connaissances primordiales des êtres humains; il cherche ensuite à définir la didactique, en extension partielle, au moyen de ses théories et concepts qui peuvent le plus contribuer à tenir compte de ces connaissances primordiales ; il présente enfin certaines menaces qui pèsent sur la didactique et sont également une façon de définir la didactique par la négative.

\section{Les connaissances primordiales et la didactique}

Précédée par de nombreuses espèces du genre Homo, notre espèce, Homo sapiens, est apparue il y a environ 200000 ans. Pendant plus de $95 \%$ de tout ce temps, les êtres humains ont été des chasseurs-cueilleurs et leur cerveau a évolué pour leur permettre de survivre dans un environnement sauvage, et souvent hostile, où ils devaient surtout porter attention aux animaux, aux plantes, au jour et à la nuit, aux particularités du terrain, aux conditions météorologiques, à quelques objets simples et aux façons d'interagir avec leurs semblables. Or, à mesure que leur mode de vie est devenu plus sédentaire, et que les civilisations ont commencé à apparaitre, il y a environ 10000 ans, les connaissances primordiales (ou connaissances intuitives) sont devenues de moins en moins adaptées. (L'adjectif « primordial » étant employé dans le sens premier de « qui existe dès l'origine, qui est le plus ancien ».) 
Voici une liste non exhaustive, inspirée de l'ouvrage The Blank Slate de Pinker (2002), ${ }^{1}$ de ces connaissances primordiales présentées en fonction de disciplines contemporaines. On $\mathrm{y}$ reconnaitra plusieurs conceptions fréquentes chez des élèves de tout âge :

- Le langage primordial, qui comporte un lexique mental de mots et des règles grammaticales de base qui fonctionnent avec des opérateurs tels que « et », « ou », «pas », «tous» et «certains". Mais il s'agit d'un langage oral et non d'un langage écrit.

- Les mathématiques primordiales, qui renferment un système de numération primordial qui peut dénombrer de petites quantités d'objets, mais qui estime les quantités plus élevées au moyen d'approximations ; une géométrie primordiale qui, sur une surface plane, représente tous les objets en deux dimensions; et une logique primordiale qui établit spontanément un lien de cause à effet entre deux évènements qui se succèdent dans le temps.

- La géographie physique primordiale, qui sert à s'orienter et à se déplacer en se repérant à l'aide de lieux remarquables (forêt, clairière, rivière, montagne, mer, falaise, etc.). Il s'agit toutefois d'une géographie basée sur le postulat d'une Terre plate.

- L'astronomie primordiale, selon laquelle les corps célestes sont situés sur une « voute céleste». Cette voute tourne autour d'une Terre plate et comporte des « étoiles fixes » qui sont immobiles les unes par rapport aux autres, et des « astres errants » (exemple : les planètes) qui se déplacent parmi les astres fixes.

- La biologie primordiale, en vertu de laquelle les plantes et les animaux, qui ont toujours été les mêmes, possèdent une « essence » ou un «principe » invisible qui les maintient en vie.

- La physique primordiale, qui affirme que les objets lourds tombent plus vite que les objets légers et que les projectiles se déplacent grâce à une « impulsion » qui se dissipe peu à peu.

- La chimie primordiale, qui dit que la friction peut créer du feu, mais que la combustion fait disparaitre la plus grande partie de la matière.

- L'ingénierie primordiale, qui postule que la façon dont fonctionne un système (exemple : un arc et des flèches) découle nécessairement des intentions de son concepteur et de la façon dont il s'en sert. Il ne peut donc pas exister de systèmes

\footnotetext{
${ }^{1}$ Cet ouvrage a été traduit en français sous le titre «Comprendre la nature humaine », aux éditions Odile
} Jacob (2005). Il est maintenant considéré comme un classique sur le thème du débat entre l'inné et l'acquis. 
très complexes (exemples : un être vivant, un orage, un volcan) sans concepteur ou opérateur surnaturel.

- L'histoire primordiale, transmise de façon orale, qui est un ensemble de récits mythiques.

- L'art primordial, qui peut viser, entre autres, à orner (exemple : collier de coquillages), à affirmer une présence (exemple : contour d'une main sur un rocher) ou à établir un lien avec le monde surnaturel (exemple : totem), mais qui est toujours relativement figuratif ou géométrique.

- La psychologie primordiale, pour laquelle les autres êtres humains ne sont pas des objets, des plantes ou des animaux, mais des individus animés par une «âme ». Les croyances et les désirs de cette âme sont les causes des comportements.

On constate donc que, dans de nombreux domaines du savoir, l'évolution n'a pas donné au cerveau de l'Homo sapiens les perceptions et les connaissances qui seraient adaptées au monde actuel. Par conséquent, les savoirs contemporains sont presque contre nature pour les êtres humains. Malheureusement, ni les spécialistes des diverses disciplines ni les pédagogues ne tiennent compte du fait que le cerveau de l'élève doit constamment acquérir des concepts et résoudre des problèmes auxquels l'évolution ne l'a pas préparé.

Seule la didactique a compris à quel point il faut faire preuve d'ingéniosité pour que l'élève puisse construire des connaissances modernes sur une fondation de connaissances primordiales qui, même si elles seront toujours présentes dans son cerveau, ne sont pas celles dont il aura besoin pour réussir à l'école et dans la vie.

Pour ce faire, la didactique, en s'appuyant notamment sur l'épistémologie, a développé un grand nombre de théories et de concepts particulièrement utiles pour les enseignants, les parents, et tous ceux qui sont concernés par l'acquisition de connaissances modernes par les élèves.

\section{La méthode de sélection et d'analyse des théories et concepts de la didactique}

Définir un domaine ou une discipline en extension consiste à en présenter toutes les théories et tous les concepts (Comte-Sponville, 2013). Cet article n'a pas la prétention de définir la didactique en extension complète. Il repose plutôt sur une méthode d'analyse de contenu (Bardin, 2003) qui place l'accent sur les théories et les concepts les plus utiles dans une perspective évolutionniste, c'est-à-dire sur les théories et concepts directement en lien avec la prise en compte des connaissances primordiales des élèves. Par exemple, selon cette perspective, les concepts de transposition didactique et de changement conceptuel sont 
indispensables, tandis que ceux de conscience disciplinaire ou de pratiques sociales de références sont plutôt secondaires et n'ont pas été retenus. Dans cet article, la didactique est donc définie en extension partielle.

De plus, ces théories et concepts sont analysés principalement à la lumière de la façon dont ils peuvent être appliqués pour tenir compte de ces connaissances primordiales dans l'enseignement et l'apprentissage des disciplines scolaires. Par exemple, tel que déjà mentionné, les conceptions fréquentes des élèves, dans plusieurs disciplines, sont souvent étrangement semblables aux connaissances primordiales présentées précédemment. L'analyse a également permis de dégager certains liens essentiels entre les connaissances primordiales et la raison d'être de plusieurs autres théories et concepts de la didactique.

Les théories et concepts retenus proviennent d'ouvrages de référence bien connus, tels que le Dictionnaire des concepts fondamentaux des didactique de Reuter (2013), La didactique en questions de Cornu et Vergnioux (1992) ainsi que d'ouvrages plus spécialisés tels que Mots-clés de la didactique des sciences d'Astolfi, Darot, Ginsburger-Vogel et Toussaint (2008) et d'autres qui sont mentionnés dans le texte. Ces ouvrages sont parmi les plus souvent consultés et cités lorsqu'il s'agit de définir des théories et concepts de la didactique.

Enfin, vers la fin de l'article, dans la section intitulée «La didactique menacée », la didactique est définie par la négative, au moyen de ce en quoi elle ne consiste pas ou ne devrait pas consister.

\section{Quelques théories et concepts de la didactique}

La didactique est née, au début des années 1970, d'une volonté de constituer un domaine de recherche autonome 1) par rapport aux disciplines scientifiques et 2) par rapport aux autres domaines des sciences de l'éducation, notamment la pédagogie. La didactique s'oppose donc à deux visions de l'enseignement et de l'apprentissage fréquentes dans le grand public. Une vision, qu'on pourrait qualifier d'académique, selon laquelle le bon enseignant est celui qui connait sa matière. Une autre vision, qu'on pourrait qualifier de pédagogique, selon laquelle le bon enseignant est celui qui s'entend bien avec ses élèves et sait gérer sa classe.

Contrairement à la pédagogie, qui est à la fois l'art et la science de l'éducation et qui s'intéresse principalement aux interventions éducatives générales, la didactique se centre plutôt sur des méthodes et des situations qui sont aptes à permettre l'acquisition de connaissances dans une matière scolaire particulière. En effet, les didacticiens s'accordent 
à dire que chaque matière scolaire possède un langage et des méthodes qui lui confèrent une valeur formative particulière, et qui peut être différente de celle des autres matières scolaires (Thouin, 2014).

La didactique se caractérise donc par sa "prise en compte, incontournable, première, revendiquée, des contenus à enseigner, qui organise ses travaux » (Robert et Butlen, 2012). Il est donc souvent plus approprié de parler des didactiques, puisque la didactique d'une matière scolaire comprend des concepts, des théories et des modèles qui lui sont propres et repose sur une connaissance approfondie de la structure, de la nature et autres particularités de cette matière scolaire. Malgré tout, la plupart des didacticiens reconnaissent qu'il existe un certain nombre de notions communes à l'ensemble des didactiques et des recherches en didactique (Reuter, 2013). Plusieurs de ces notions communes sont présentées ci-après.

La suite de cette section s'articule de la façon suivante. Les grandes disciplines et théories qui forment le substrat de la didactique, soit l'épistémologie, le constructivisme et le socioconstructivisme sont d'abord présentées. Suit ensuite le triangle didactique qui annonce, dans l'ordre, ce qui relève de la relation enseignant-élève (le contrat didactique), de la relation enseignant-savoir (la transposition didactique), puis ce qui relève de la relation élève-savoir (exemples : conception des élèves, changement conceptuel, démarche didactique, erreurs). Elle se termine par quelques considérations générales au sujet de l'école et de la culture.

\section{L'épistémologie}

L'épistémologie n'est évidemment pas un concept de la didactique, mais il s'agit d'une discipline contributoire d'une telle importance qu'il vaut la peine de la présenter brièvement.

Le terme épistémologie provient de deux mots grecs, épistémè, qui signifie connaissance ou science, et logos, qui signifie langage, étude scientifique, ainsi que discours et jugement (et qui se retrouve également dans des termes tels que biologie et géologie). Plus précisément, l'épistémologie s'intéresse à la définition, au sens et à la légitimité des disciplines scientifiques, à l'élaboration et à la comparaison de diverses conceptions de la recherche, aux langages des sciences ainsi qu'à la nature des problèmes scientifiques.

Il est souvent question d'épistémologie, en didactique, parce que cette dernière s'intéresse à la nature et à la structure conceptuelle des savoirs de chacune des disciplines enseignées. Elle s'intéresse également, depuis les travaux de Jean Piaget en particulier, à la construction des savoirs, un domaine qu'on appelle parfois l'épistémologie génétique. Il est aussi question d'épistémologie, en recherche, parce que celle-ci a pour but de produire des 
savoirs et que l'épistémologie fournit des critères permettant de juger de la scientificité de ces savoirs et des méthodes employées pour les produire.

À titre d'exemple, voici quelques questions de nature épistémologique dans diverses disciplines :

- Français langue première: Une langue est-elle surtout un outil utile à la communication entre les personnes ou un véhicule de la pensée qui serait indispensable même si chaque être humain vivait seul sur une ile déserte?

- Mathématiques : Un théorème mathématique, comme le théorème de Pythagore qui exprime la relation entre la longueur de l'hypoténuse d'un triangle rectangle et celles des deux autres côtés, a-t-il été découvert ou construit?

- Sciences: Une expérience scientifique peut-elle prouver qu'une hypothèse est vraie?

- Histoire : L'histoire est-elle principalement un ensemble de récits ?

- Arts visuels : Les graffitis et les «tags » peuvent-ils être considérés comme de l'art, au même titre que certaines œuvres d'art contemporain?

L'étude de la construction des savoirs permet notamment de comprendre comment les savoirs se sont graduellement éloignés des connaissances primordiales.

\section{Le constructivisme}

Depuis environ une vingtaine d'années, le terme constructivisme est d'usage courant dans divers textes en sciences de l'éducation, mais le sens qu'on lui donne est loin d'être univoque. Au pire, il signifie simplement toute conception de l'enseignement et de l'apprentissage qui s'éloigne des exposés magistraux et de la mémorisation. Une bonne façon d'éviter ce genre de caricature est de faire la distinction, proposée par Jean-Pierre Astolfi et ses collègues (2008), entre les constructivismes épistémologique, psychologique et didactique.

Le constructivisme épistémologique, qui met l'accent sur le caractère construit des savoirs, s'oppose à l'empirisme ou au positivisme basé sur des « faits ». Selon le constructivisme épistémologique, les savoirs ne sont pas des faits indiscutables mais des propositions, éventuellement réfutables, de réponses à des questions. Tout concept, qu'il s'agisse du concept de mot, de nombre, de cellule, ou de démocratie n'est pas réel, mais construit. Il importe toutefois de préciser que le constructivisme épistémologique, surtout dans ses versions les plus radicales, est plutôt mal accepté des épistémologues, notamment parce 
qu'il peut facilement mener à de sérieuses dérives, par exemple à un relativisme excessif selon lequel toute théorie qui fonctionne pourrait être considérée comme acceptable.

Le constructivisme psychologique, qui découle des travaux de Jean Piaget, s'oppose au béhaviorisme. D'après le constructivisme psychologique, apprendre n'est pas simplement acquérir des comportements, ce qui pourrait relever du dressage, mais construire et structurer des connaissances. Cette construction et cette structuration dépendent souvent des déséquilibres qui découlent des conflits cognitifs que vit l'élève. Ces conflits, vécus de façon individuelle par l'élève, sont appelés des conflits de centration parce que l'élève, pour retrouver son équilibre cognitif, doit se recentrer sur une nouvelle structure conceptuelle.

Le constructivisme didactique s'oppose au dogmatisme et à l'enseignement transmissif. Enseigner n'est pas seulement présenter le savoir, mais construire des dispositifs didactiques, des situations d'enseignement et d'apprentissage qui facilitent la construction de connaissances par l'élève et l'aident à les distinguer de certaines connaissances primordiales.

\section{Le socioconstructivisme}

Lorsque l'accent est placé sur les conflits sociocognitifs plutôt que sur les conflits de centration ainsi que sur les interactions vécues par les élèves entre eux et avec leur milieu, on parle alors de socioconstructivisme (Vygotsky, 1934/1997) plutôt que de constructivisme. Ces interactions sont souvent d'autant plus dynamiques qu'elles portent en partie sur des connaissances primordiales, mais fausses, auxquelles certains élèves sont très attachés.

Le socioconstructivisme possède également des versions controversées selon lesquelles, par exemple, toute science, ou toute théorie, n'est qu'une construction sociale, une production culturelle plus ou moins arbitraire. Depuis une vingtaine d'années, des centaines d'ouvrages, d'articles et de thèses portent un titre qui commence par « La construction sociale de... », incluant, par exemple, « La construction sociale des sciences », « La construction sociale de l'école », « La construction sociale de l'hyperactivité », etc. La controverse vient du relativisme excessif avec lequel les « constructions sociales » sont présentées, comme si tout ce qui serait théoriquement possible était équivalent, comme si tout se valait.

Mais, comme l'a déjà fait remarquer, à la blague, le biologiste Richard Dawkins, personne n'est socioconstructiviste (au sens de construction sociale) quand dans un avion, à 10000 mètres d'altitude, il y a de fortes turbulences : tous les passagers souhaitent que les théories 
de la physique et de l'aéronautique soient vraies et que la portance des ailes de l'avion soit bien réelle.

\section{Le triangle didactique et le rapport au savoir}

Tel que proposé par le didacticien français Yves Chevallard (1985/1991), un système didactique d'enseignement et d'apprentissage peut être représenté par un triangle dont les sommets sont les savoirs, l'élève et l'enseignant.

Les sommets de ce triangle didactique, considérés seuls, concernent des domaines de recherche qui ne sont pas liés à la didactique. L'étude des savoirs relève des diverses disciplines. L'étude de l'élève et des modèles d'apprentissage ainsi que de l'enseignant et des modèles d'enseignement appartiennent à la pédagogie. Les domaines de recherche propres à la didactique sont représentés par les trois côtés de ce triangle, à savoir les relations entre deux des trois sommets que sont l'enseignant, l'élève et les savoirs.

La relation entre l'enseignant et le savoir. C'est le domaine de l'élaboration des contenus d'enseignement. La didactique s'intéresse particulièrement aux modifications, appelées transposition didactique, que subissent les savoirs savants (c'est-à-dire les savoirs produits par la communauté scientifique) afin de devenir des savoirs scolaires. Elle s'intéresse aussi au matériel didactique, qui est l'ensemble des aides didactiques, documents et outils qui sont les résultats de cette transposition.

La relation entre l'enseignant et l'élève. C'est le domaine, entre autres, du contrat didactique entre l'enseignant et l'élève, et qui désigne l'ensemble implicite des obligations réciproques entre l'enseignant et l'élève en ce qui concerne les savoirs. Chacun a des droits et des responsabilités qui sont rarement énoncés mais qui font partie des rôles de l'enseignant et de l'élève : en proposant une activité à un élève, l'enseignant a des attentes à l'égard de celui-ci mais ne les énonce pas toujours; de son côté, l'élève sait que l'enseignant a certaines attentes et il essaie d'y répondre au meilleur de sa compréhension. L'évaluation des apprentissages, par exemple, bien qu'elle relève aussi de la relation entre l'élève et le savoir, est un aspect important du contrat didactique, car elle confère des responsabilités importantes, qui doivent être correctement perçues de part et d'autre, à l'enseignant et à l'élève.

La relation entre l'élève et le savoir. Ce domaine est celui du rapport de l'élève au savoir. En d'autres termes, c'est le domaine des stratégies qui permettront à l'élève de s'approprier des savoirs (qui lui sont extérieurs) pour en faire des connaissances (qui lui sont propres). La didactique, qui ne considère pas l'élève comme une "boite vide » ou une " tabula rasa », s'intéresse aux façons dont les élèves arrivent à construire leurs nouvelles 
connaissances sur la base des connaissances primordiales, ou conceptions initiales, qui constituent souvent des obstacles à l'apprentissage, mais qui peuvent parfois, au contraire, jouer le rôle d'adjuvants dans l'acquisition de certains concepts. La conception et l'analyse d'une démarche didactique qui permet aux élèves de faire des apprentissages sont des thèmes de recherche fondamentaux en didactique. Un des objectifs essentiels de toute didactique est de faire en sorte que l'élève développe un rapport harmonieux au savoir.

En didactique, les trois relations (enseignant-savoir, enseignant-élève et élève-savoir) doivent être considérées. Une attention exclusive accordée à la relation entre l'enseignant et le savoir risque de conduire à une pédagogie encyclopédique peu stimulante. Une attention exclusive accordée à la relation entre l'enseignant et l'élève risque de mener à une pédagogie sociale qui néglige les contenus à apprendre. Une attention exclusive accordée à la relation entre l'élève et le savoir risque de se traduire par une pédagogie exploratoire qui manque d'encadrement puisque l'enseignant a disparu.

Le milieu. Pour compléter le triangle didactique, il est souvent utile d'imaginer que le support (feuille de papier, écran d'ordinateur) sur lequel le triangle est dessiné représente le milieu dans lequel se vivent les interactions enseignant-savoir, enseignant-élève et élèvesavoir. Dans un grand nombre de recherches en didactique ce milieu est une classe, mais il est souvent nécessaire d'en préciser les caractéristiques : une classe régulière, une classe d'élèves doués, une classe d'adaptation scolaire, une classe d'accueil pour élèves de familles d'immigration récente, etc. Dans d'autres recherches en didactique, le milieu sera une bibliothèque, un musée, un centre de loisir, etc. Un changement de milieu entraine des modifications importantes à de nombreuses facettes d'un système didactique, par exemple, des relations différentes entre l'enseignant et l'élève, l'enseignant et le savoir ou l'élève et le savoir.

La sous-section suivante apporte des précisions au sujet des savoirs, qui se trouvent à un des sommets du triangle didactique.

\section{Les savoirs et les connaissances}

Bien que les mots savoirs et connaissances soient souvent employés comme synonymes, leur sens, en didactique, est différent (Reuter, 2013).

Les savoirs, qui sont les résultats des travaux d'une communauté scientifique, désignent un ensemble de concepts, de lois, de modèles et de théories institués et dépersonnalisés. Habituellement, les scientifiques de la discipline qui maitrisent ces savoirs et les autorités 
qui décident de les retenir pour un programme d'études s'entendent sur les mêmes définitions et formulations de ces savoirs.

Les connaissances sont les résultats, intériorisés par un élève en fonction de son expérience et de ses représentations, de son apprentissage de savoirs. En théorie, on peut donc se retrouver avec autant d'ensembles de connaissances qu'il y a d'élèves dans une classe, puisque les connaissances sont le résultat d'une interprétation et d'une compréhension subjectives et partielles de savoirs. L'interférence entre les savoirs enseignés et les connaissances primordiales entraine des incohérences inévitables, qui pourront éventuellement être corrigées via une transposition didactique adéquate, un concept présenté plus loin.

La sous-section suivante aborde la relation entre l'enseignant et ses élèves.

\section{Le contrat didactique}

Certains professeurs, surtout au niveau universitaire, appliquent une «pédagogie du contrat » avec leurs étudiants. Cette façon de procéder, qui peut prendre la forme d'un contrat écrit, consiste pour le professeur et chacun de ses étudiants à s'entendre sur les attentes qui correspondent aux divers résultats possibles. Par exemple, l'étudiant qui opte pour un résultat élevé s'engage à effectuer des travaux d'une qualité plus grande que celui qui opte pour un résultat moyen, par exemple parce que son travail comporte plus de références, est mieux structuré ou est mieux écrit.

La notion de contrat didactique, d'abord proposée par Guy Brousseau (1986) en didactique des mathématiques, est très différente de ce type de contrat explicite. Le contrat didactique désigne les comportements de l'enseignant attendus par les élèves (exemple : expliquer clairement les notions les plus importantes), les comportements de l'élève attendus par l'enseignant (exemple : décrire la démarche ou le raisonnement et pas seulement la réponse finale à un exercice ou un problème) ainsi que les rapports de l'enseignant et de l'élève au savoir visé par l'apprentissage (exemple : on présume que l'enseignant maitrise davantage le savoir que l'élève, mais qu'il ne se contente pas de l'imposer de façon dogmatique).

La réussite des élèves dépend, dans une large mesure, du respect des termes du contrat didactique. Par conséquent, la didactique s'intéresse particulièrement à tout ce qui peut entrainer une rupture du contrat et aux façons d'éviter ces ruptures. Par exemple, le fait, pour un enseignant, d'enseigner presque mot pour mot les réponses aux questions habituellement posées lors des examens officiels constituerait une rupture du contrat 
puisqu'une telle pratique dévaloriserait la responsabilité de la construction du savoir par l'élève et le transformerait en simple « perroquet ».

On peut donc dire, de façon générale, que le contrat didactique définit le métier d'élève tout autant qu'il définit la profession enseignante. Une école où les enseignants et les élèves refuseraient de jouer leur rôle et d'assumer leurs responsabilités finirait vite par s'effondrer. À ce sujet, un des rôles importants des élèves est d'apprendre à remettre en question leurs intuitions premières, qui reposent sur des connaissances primordiales.

La prochaine sous-section traite de la relation entre l'enseignant et le savoir.

\section{La transposition didactique}

Les savoirs enseignés à tous les ordres d'enseignement sont bien différents des savoirs produits récemment par la communauté scientifique. En effet, les concepts et les théories enseignés aux élèves ne sont pas seulement des simplifications des concepts et des théories de diverses disciplines, mais le résultat de reconstructions qui les modifient et transposent le savoir savant en savoir scolaire. Cette reconstruction porte le nom de transposition didactique (Chevallard, 1985/1991).

Les deux principaux processus qui interviennent, dans cette transposition, sont la sélection et la transformation des savoirs. Cette sélection et cette transformation sont d'abord effectuées par les auteurs de programmes, de manuels scolaires et de matériel didactique, ce qui constitue la transposition didactique externe. Elle est aussi effectuée par les enseignants, qui adaptent les programmes, les manuels scolaires et le matériel didactique pour leurs élèves, ce qui constitue la transposition didactique interne. Dans certains cas, la transposition didactique peut même aboutir à des savoirs scolaires qui n'ont plus d'équivalent dans le savoir scientifique moderne (exemples : la géométrie euclidienne, le modèle planétaire de l'atome).

La sélection des savoirs. Il est impossible d'enseigner toute une discipline. Il faut donc faire certains choix en fonction, par exemple, des buts poursuivis par le système éducatif et le programme de formation. Il est évident, par ailleurs, que certains concepts jugés trop difficiles (exemple : les temps composés du subjonctif, en français) ne sont pas abordés à certains niveaux et, à l'inverse, que certains concepts de base qui ne sont plus étudiés par des spécialistes (exemple: la notation de Lewis des électrons de valence, en chimie) peuvent constituer des aspects importants du programme. 
La transformation des savoirs. Les conditions dans lesquelles le savoir est transmis sont différentes de celles dans lesquelles il est produit et entrainent une structuration particulière. Par conséquent, les savoirs scolaires ne sont pas organisés de la même façon que les savoirs savants. Les problèmes abordés avec les élèves et les relations établies entre les concepts enseignés diffèrent des problèmes et des relations qui préoccupent les scientifiques. Cette transformation des savoirs savants en savoirs scolaires peut prendre une ou plusieurs des formes suivantes :

- La dogmatisation: Donne l'impression que les savoirs sont immuables et irréfutables.

- La décontextualisation : N'établit pas de liens entre les savoirs,

- La dépersonnalisation: Ne présente pas les scientifiques à l'origine des savoirs.

- La désyncrétisation : Fait perdre sa cohérence au savoir scientifique.

- La programmation : Réorganise le savoir selon une logique scolaire.

- La reformulation : Reformule le savoir sous forme de compétences, objectifs, etc.

- L'opérationnalisation : Place l'accent sur les savoirs qui peuvent être évalués.

La sélection et la transformation des savoirs qui caractérisent les transpositions didactiques sont inévitables. Il serait impossible d'enseigner directement les savoirs de la communauté scientifique. Mais certaines transpositions didactiques sont mieux réussies que d'autres, particulièrement lorsqu'elles évitent une décontextualisation, une dogmatisation, une dépersonnalisation, une désyncrétisation et une opérationnalisation trop radicales des savoirs. Les meilleures transpositions didactiques sont souvent celles qui tiennent compte, d'une façon ou d'une autre, du fossé qui sépare les connaissances primordiales des connaissances attendues.

La sous-section suivante présente des concepts qui relèvent de la relation élève-savoir.

\section{Les conceptions des élèves et les obstacles épistémologiques}

Contrairement à l'idée longtemps tenue pour évidente, l'élève, comme l'ont montré Bachelard (1934/1993) et Migne (1969), n'arrive pas en classe la tête vide mais possède déjà une foule de conceptions (parfois appelées représentations) au sujet des concepts à l'étude. Ces conceptions, qui peuvent souvent être prédites à partir des connaissances primordiales dont il a été question précédemment, ont tendance à résister à l'enseignement et à persister longtemps. 
Plusieurs de ces conceptions sont invariantes, et ont été répertoriées (exemples : le Soleil tourne autour de la Terre; la multiplication de deux nombres donne toujours un nombre plus grand que ceux qui sont multipliés; les verbes au subjonctif existent dans toutes les langues), mais d'autres sont variables en fonction du contexte de production (exemple : une marche en forêt nous oxygène mais il ne faut pas laisser de plantes dans la chambre d'un malade).

Dans certaines disciplines, les conceptions peuvent être regroupés en grandes catégories que Bachelard appelait des obstacles épistémologiques. Par exemple, l'obstacle verbal consiste à donner une explication au moyen de mots qui n'expliquent rien (exemple : l'opium fait dormir parce qu'il contient un principe dormitif) et l'obstacle substantialiste consiste à expliquer un phénomène au moyen d'une substance (exemple : la chaleur est une substance qui se déplace des objets chauds vers les objets froids).

\section{Les modèles de changement conceptuel}

Corollaire du point précédent, la didactique a proposé divers modèles qui expliquent l'évolution des conceptions des élèves. Comme Potvin (2018) l'a clairement montré, on peut classer les modèles de changement conceptuel en trois grandes catégories dont l'ordre correspond à leur évolution historique : 1) les modèles de remplacement des conceptions, 2) les modèles de transformation des conceptions et 3 ) les modèles de coexistence des conceptions.

Les modèles de remplacement des conceptions sont basés sur le postulat que l'enseignement efficace d'une discipline scolaire permet de remplacer les conceptions des élèves par les concepts de la communauté scientifique. Le plus connu de ces modèles est celui de Posner, Strike, Hewson et Gertzog (1982) selon lequel le changement conceptuel peut se produire si l'élève vit des conflits cognitifs (exemple : une démonstration dont les résultats sont surprenants) qui lui permettent de ressentir une insatisfaction envers ses conceptions initiales, et que, par la suite, l'élève trouve que les concepts par lesquels l'enseignant souhaite que l'élève remplace ses conceptions sont intelligibles, plausibles et fertiles. Bien que, de nos jours, les modèles de remplacement soient encore les plus populaires, des recherches plus récentes ont montré que les conflits cognitifs sont rarement aussi efficaces que ces modèles le prévoyaient.

Les modèles de transformation des conceptions reposent sur l'idée que le changement conceptuel est plutôt une restructuration des structures cognitives des élèves. Dans ce contexte, on considère les conflits cognitifs peuvent s'avérer utiles, mais que leur visée est plus modeste et consiste principalement à modifier les catégories dans lesquelles les élèves 
placent divers objets et phénomènes. Le modèle des p-prims («primitives phénoménologiques ») de diSessa (1993), qui décrit certaines interprétations élémentaires fréquentes, telle que " plus près = plus fort », et le modèle des catégories ontologiques de Vosniadou et Brewer (1992), qui décrit des caractéristiques implicites d'objets ou d'environnements, tel le fait que la chute d'un objet se produit toujours vers le bas, sont deux exemples représentatifs des modèles de transformation des conceptions. Toutefois, certaines recherches contemporaines font ressortir le fait que les modèles de transformation des conceptions expliquent mal la persistance de certaines conceptions chez les élèves.

Les modèles de coexistence des conceptions, qui s'appuient en partie sur des résultats de recherche en neurosciences, proposent l'idée que les conceptions initiales persistent, après le changement conceptuel, et que le cerveau de l'élève doit les inhiber pour être en mesure de faire appel aux concepts scientifiques appris en classe. Le principe de l'utilité cognitive perçue (Ohlsson, 2009), selon lequel des idées peuvent être reconnues comme plus puissantes par les élèves, pourrait expliquer comment des concepts scientifiques réussissent à inhiber certaines conceptions. Un exemple très représentatif de modèle de coexistence des conceptions est le modèle de la prévalence conceptuelle (Potvin, Sauriol, Riopel, 2015). Selon ce modèle, il est préférable de commencer l'enseignement par la présentation des concepts à faire apprendre, de développer ensuite chez les élèves le réflexe d'inhiber leurs conceptions non scientifiques, puis d'automatiser, dans divers contextes, l'emploi des concepts enseignés.

\section{La démarche didactique}

La démarche didactique est constituée des séquences et des situations qui permettent à l'élève de faire des apprentissages, c'est-à-dire, dans bien des cas, de passer de connaissances primordiales à des connaissances plus modernes.

Les séquences didactiques sont les ensembles d'activités portant sur les mêmes thèmes, concepts ou notions. Par exemple, une séquence comporte souvent des activités fonctionnelles qui peuvent servir de mise en situation, des activités de résolution de problème, des activités de structuration qui peuvent permettre une intégration des connaissances, et des activités d'enrichissement. Les situations didactiques sont les éléments ou les ensembles d'éléments nécessaires au déroulement des séquences didactiques (exemples : projet, document, problème, exercice, etc.).

L'ingénierie didactique est le volet pratique de la didactique qui porte principalement sur la conception de séquences didactiques, de situations didactiques et d'activités 
d'enseignement et d'apprentissage. L'ingénierie didactique est également un type de recherche de développement particulièrement important en didactique (Artigue, 1996).

Dans la sous-section suivante, il est question de deux types fréquents de situations didactiques.

\section{Les situations-problèmes et les problèmes ouverts}

Dans plusieurs disciplines, une situation-problème (De Vecchi, 2007) ou un problème ouvert (Boilevin, 2013) est l'élément-clé d'une séquence didactique. Idéalement, un bon problème :

- Amène les élèves à remettre en question certaines de leurs conceptions, représentations initiales ou connaissances primordiales ;

- Leur permet de jouer un rôle actif dans leur apprentissage ;

- Est perçu par les élèves comme un défi, une énigme, ce qui permet la dévolution (c'est-à-dire l'engagement de l'élève) ;

- Se situe dans la zone de développement proximale de l'élève (ce qui signifie que la situation problème n'est ni trop facile, ni trop difficile) et permet une bonne mobilisation intellectuelle;

- Comporte diverses solutions ou permet diverses approches ;

- Prévoit des échanges entre les élèves ;

- Conduit les élèves à une évolution de leurs conceptions.

\section{Les concepts}

En didactique, les concepts s'inscrivent dans un champ conceptuel et une trame conceptuelle.

Les concepts sont des représentations mentales générales et abstraites permettant d'organiser et de simplifier les perceptions et les connaissances. Par exemple, les mots « cellule » (biologie), «température » (physique), « siècle» (histoire), "phrase » (français) et «fonction» (mathématiques) désignent des concepts. Un même mot, par exemple le mot «chaleur», peut parfois désigner à la fois un concept scientifique (un concept employé par des physiciens), un concept scolaire (un concept scientifique qui se retrouve sous une forme simplifiée dans un programme de formation) et un concept quotidien (un concept de la vie de tous les jours), ce qui peut être la cause de confusions, 
parce que le sens exact du concept diffère selon la sphère de pratique dans laquelle il est employé.

Le champ conceptuel, une notion proposée par Gérard Vergnaud (1990), permet de préciser la notion de concept scolaire. Un champ conceptuel est un ensemble de situations, de formes langagières et d'invariants opératoires qui donnent du sens à des concepts scolaires. Par exemple, les exercices portant sur l'addition ou la soustraction, ou sur l'accord des adjectifs, au primaire, se traduisent habituellement par des situations, des consignes et des échanges avec les élèves (formes langagières) ainsi que par des conduites et des comportements des élèves relativement fixes (invariants opératoires). Un champ conceptuel comporte donc des aspects explicites (exemple : langage utilisé) et des aspects implicites (exemple : conduite des élèves).

Les trames conceptuelles (appelés aussi réseaux notionnels) sont des diagrammes formés de figures géométriques reliées par des segments de droite ou des flèches qui illustrent les liens entre des concepts. Une trame conceptuelle peut être un outil intéressant pour dresser un portrait de l'état des connaissances des apprenants, incluant leurs conceptions (ou connaissances primordiales), tout comme il peut être utile au concepteur de programme et à l'enseignant qui souhaitent situer des concepts dans un cadre plus large.

\section{Les niveaux de formulation des concepts}

Contrairement à plusieurs concepts du langage courant, un concept scientifique ou scolaire peut être formulé à divers niveaux (Develay, 1991). L'enseignant et l'élève doivent savoir utiliser le niveau de formulation approprié dans une situation donnée.

Par exemple, il n'est pas très utile à un automobiliste en panne sèche de savoir que le concept «essence » peut être défini comme un liquide pétrolier artificiellement coloré distillant à $200{ }^{\circ} \mathrm{C}$. Savoir qu'il s'agit d'un carburant est bien suffisant. Comme autres exemples, les concepts de « respiration » (biologie) et de «fonction » (mathématiques) se complexifient au fil de la progression d'un niveau scolaire au suivant.

Les niveaux de formulation se distinguent sur le plan langagier (terminologie plus ou moins spécialisée pour définir le concept à chaque niveau), psychogénétique (développement intellectuel plus ou moins avancé requis pour comprendre le concept à chaque niveau) et épistémologique (conception de l'activité scientifique plus ou moi élaborée sur laquelle repose la construction du concept à chaque niveau). 
Cela dit, tout niveau de formulation d'un concept, même le plus élémentaire, permet de distinguer ce concept des connaissances primordiales qui sont souvent celles des élèves.

\section{Les erreurs}

Comme l'a bien montré Astolfi (1997), les erreurs des élèves, qui ont longtemps été considérées de façon négative, sont maintenant abordées, en didactique, comme des indices pour repérer leurs difficultés et comprendre le processus d'apprentissage et peuvent véritablement devenir un « outil pour enseigner ».

Voici un bref aperçu de divers types d'erreurs et des façons d'aider l'élève à les surmonter :

- Les erreurs liées aux conceptions fréquentes (mais fausses). Plusieurs erreurs des élèves s'expliquent par leurs conceptions ou connaissances primordiales ainsi que par les obstacles épistémologiques dans lesquelles elles peuvent être regroupées. Proposer des situations qui feront vivre des conflits cognitifs et sociocognitifs aux élèves pourra favoriser l'inhibition de leurs conceptions et le recours à des concepts plus scientifiques.

- Les erreurs liées à la nouveauté des termes et des symboles. Les élèves comprennent souvent mal le sens de certains termes et symboles qui sont évidents pour un enseignant qui connait bien la discipline dans laquelle ils sont employés. Certaines recherches ont montré que les élèves doivent avoir vu et utilisé des dizaines de fois des termes et symboles nouveaux avant qu'ils ne fassent vraiment partie de leur vocabulaire.

- Les erreurs liées au niveau d'abstraction du contenu. Des concepts tels que celui de « déterminant» (français), de «fonction » (mathématiques), d' « énergie » (sciences) ou de «démocratie» (univers social) sont très abstraits et ne se construisent que graduellement. La progression qui mène à leur compréhension doit être conçue par étapes bien étagées.

- Les erreurs liées à une mauvaise transposition didactique. Les transpositions didactiques qui décontextualisent, dogmatisent, dépersonnalisent, désyncrétisent et opérationnalisent les savoirs de façon trop radicale peuvent être sources d'erreurs, entre autres parce qu'elles rendent une discipline scolaire moins cohérente, stimulante et intelligible.

- Les erreurs liées aux obstacles didactiques. Les obstacles didactiques sont causés par l'enseignement. Recourir à une analogie, donner un exemple, employer une métaphore comportent toujours le risque d'entrainer de fausses interprétations. 
- Les erreurs liées aux écarts aux démarches attendues. Certains élèves commettent des erreurs parce qu'ils ne procèdent pas de la façon qui leur a été enseignée pour résoudre un problème mathématique, corriger un texte, ou réaliser une expérience de laboratoire. Sans perdre de vue l'utilité de la démarche usuelle, il est intéressant d'analyser, avec les élèves, dans quelle mesure une autre démarche peut conduire à un résultat équivalent, ce qui contribue à développer leur créativité et leur esprit d'initiative.

- Les erreurs liées à la compréhension des consignes. Plusieurs erreurs découlent du fait que les élèves ne comprennent pas bien ce qu'on leur demande, surtout quand des consignes leur sont présentées par écrit. Dans certains cas, la lisibilité des textes destinés aux élèves n'est pas appropriée pour leur niveau. Dans d'autres cas, les élèves n'ont pas eu assez d'occasions de s'habituer à interpréter correctement le langage propre à ce type de textes.

- Les erreurs liées au contrat didactique. L'ensemble implicite des droits et des responsabilités qui définit les rôles de l'enseignant et des élèves est parfois mal décodé par les élèves, ce qui nécessite alors de le rendre plus explicite, surtout pour que les élèves évitent de développer des habitudes incompatibles avec les pratiques en usage dans chacune des disciplines, par exemple lorsque l'enseignant doit évaluer les apprentissages.

- Les erreurs liées à la conception des situations. Les situations didactiques ne sont pas toujours bien adaptées aux élèves. Pour favoriser les apprentissages escomptés, une situation didactique doit être claire, pertinente et bien structurée. Elle doit aussi, comme l'a bien montré Vygotsky (1985), se situer dans une zone de développement proximal, c'est-à-dire être assez difficile pour constituer un défi stimulant sans toutefois être ardue au point d'être perçue comme insurmontable.

\section{La dévolution et la contextualisation}

Une séquence didactique comporte souvent une phase de dévolution et de contextualisation, suivie d'une phase de décontextualisation et d'institutionnalisation (Brousseau 1998).

La dévolution désigne l'ensemble des moyens par lesquels l'enseignant suscite l'engagement et la mobilisation des élèves. Concrètement, elle s'effectue en proposant des défis, énigmes, problèmes et autre activités fortement contextualisées (en lien avec des situations courantes de la vie des élèves), dans lesquels les élèves se mettent à réaliser les limites et les lacunes de leurs connaissances primordiales et se sentent véritablement concernés. Lorsque l'intention d'enseigner disparait au profit de cette dévolution, la 
situation devient temporairement a-didactique (c'est-à-dire que son intention didactique est oubliée pendant quelque temps), puisque l'élève s'approprie la tâche et s'implique au point de ne plus la considérer comme une exigence scolaire ou même, dans le meilleur des cas, de ne pas se préoccuper de l'intention d'enseignement et d'apprentissage qui justifie le choix de cette situation par l'enseignant.

\section{La décontextualistation et l'institutionnalisation}

Dans une séquence didactique, la phase de décontextualisation et d'institutionnalisation suit normalement celle de la dévolution et de la contextualisation. La décontextualisation consiste à sortir les apprentissages du contexte particulier ayant permis l'engagement de l'élève et à les situer dans l'ensemble des savoirs formels, souvent abstraits. Ces savoirs formels sont aussi des savoirs institutionnels, c'est-à-dire prescrits par les programmes de formation, ce qui explique qu'une institutionnalisation (c'est-à-dire une formulation dans les termes des programmes officiels) accompagne cette décontextualisation.

En pratique, la décontextualisation et l'institutionnalisation se vivent souvent sous la forme d'activités de structuration (exemple: écriture d'un texte, préparation d'un exposé, construction d'un réseau notionnel, remise d'un rapport, etc.) qui permettent aux élèves de faire la synthèse des savoirs essentiels de la séquence didactique et sont des occasions pour l'enseignant de faire ressortir le fossé qui sépare leurs connaissances primordiales des connaissances scientifiques à acquérir. C'est une phase qui, faute de temps en classe, est parfois négligée, ce qui explique que certains élèves ne perçoivent pas toujours la pertinence et la cohérence de leurs apprentissages (Thouin, 2017).

\section{L'école et la culture}

L'école intéresse tout autant la pédagogie que la didactique. Mais les réflexions du philosophe français Fédier (2006) à son sujet, qui sont présentés ci-dessous, montrent bien que la culture et le savoir, comme l'a montré Gauthier (1991), sont la meilleure façon d'échapper à «l'insoutenable légèreté de la pédagogie» et de redonner son rôle fondamental à l'école. Comme nous l'avons vu, la relation entre l'élève et le savoir est au centre des préoccupations de la didactique.

Selon Fédier, les mots « école » et « scolaire », tout comme le mot anglais «school» sont dérivés du mot grec scholè. Ce mot grec, à la grande surprise de bien des gens, signifie loisir. Pourtant, quoi de plus éloigné de l'école, pour la plupart des élèves, des étudiants, des parents et des enseignants, que le loisir ? En voici l'explication. Contrairement à la conception actuelle de l'enseignement, où il s'agit avant tout de dégager les potentialités de chaque enfant et de développer chez lui des compétences afin de le diriger vers le type 
de travail dans lequel il sera le plus utile à la société, la formation, dans la civilisation gréco-romaine, est d'abord educatio, un mot latin qui désigne un itinéraire depuis la nonhumanité jusqu'à l'humanité libre. L'humanité libre se définit par la possibilité de loisir, en latin otium et en grec scholè. L'otium, la scholè, c'est la tranquillité qui permet de se livrer sans dommage à la pensée et à l'étude. Au sens étymologique, l'école est donc presque le contraire de ce qu'elle est devenue avec le temps. Ce n'est pas une corvée. C'est le loisir, la tranquillité qui donne à la pensée l'occasion de se développer en allant au contact de soi-même, des autres et du monde en toute liberté, sans l'oppression du résultat, à l'abri du matraquage de la rentabilité à tout prix.

Or c'est la culture transmise par l'école, qui comprend à la fois l'héritage du passé, la critique de cet héritage et le pouvoir de créer d'autres possibilités, qui permet le développement de la pensée. De nombreuses recherches en didactique ont d'ailleurs montré que le meilleur prédicteur de la réussite future d'un élève de la fin du primaire est sa compétence en lecture, qui elle-même dépend de sa culture, plus précisément du nombre de mots, de concepts, de lieux, d'œuvres, d'évènements et de personnages qu'il connait et dont il comprend l'importance et la signification.

\section{La didactique menacée}

La didactique est une discipline relativement nouvelle, qui compte moins de professeurschercheurs et qui est moins bien établie que d'autres domaines des sciences de l'éducation tels que la pédagogie et l'administration scolaire. En partie pour cette raison, et malgré son rôle essentiel en enseignement et en apprentissage, la didactique est constamment menacée, à la fois de l'intérieur et de l'extérieur.

En paraphrasant Milan Kundera (2000), qui parlait des petites nations, on pourrait dire, en exagérant à peine, que les petites disciplines, telles que la didactique, ne connaissent pas la sensation heureuse d'être là depuis longtemps et à jamais ; elles sont toutes passées, à tel ou tel moment de leur histoire, par l'antichambre de la mort; toujours confrontées à l'arrogante ignorance des grandes, elles voient leur existence perpétuellement menacée ou remise en question.

\section{La didactique menacée de l'intérieur}

Par exemple, certains didacticiens semblent oublier que la didactique relève du domaine cognitif et qu'elle s'intéresse principalement à la relation entre l'élève, l'enseignant et le savoir. Il leur arrive donc de se lancer dans des recherches dont les thèmes débordent du cadre de la didactique et ne se distinguent plus tellement de ceux d'autres domaines des 
sciences de l'éducation tels que la pédagogie, l'administration scolaire ou les fondements de l'éducation.

D'autres résistent mal aux sirènes de la mode (ou aux priorités souvent discutables des organismes subventionnaires) et se laissent entrainer dans des thèmes de recherche attrayants et populaires, mais qui contribuent relativement peu à des apprentissages significatifs par les élèves. L'abus du numérique, par exemple, semble nous rapprocher d'une école et d'une civilisation de l'« écran total », au sujet desquelles Baudrillard sonnait déjà l'alarme en 1998 : en français, le mot « écran » peut désigner une toile ou un appareil qui permet de voir, mais il désigne surtout un panneau ou une substance qui cache.

Enfin, d'autres didacticiens, qui préfèrent le confort de leur bureau aux contraintes du milieu scolaire et aux exigences des comités d'éthique, se réfugient dans des recherches qui, bien qu'elles relèvent de la didactique, peuvent se faire uniquement à partir de documents écrits et risquent par conséquent de les éloigner de la réalité du terrain et du " métier d'élève ». Les recherches portant sur l'analyse de manuels scolaires et de sites Web ou sur le dépouillement de statistiques de fréquentation scolaire, de résultats à des tests internationaux et de sondages en ligne entrent dans cette catégorie.

\section{La didactique menacée de l'extérieur}

Certaines universités s'avèrent peu exigeantes lorsqu'elles ouvrent des postes de professeurs-chercheurs en didactique et embauchent des candidats dont le véritable domaine de spécialisation est une autre discipline des sciences de l'éducation, voire même simplement une discipline scientifique. C'est notamment le cas lorsqu'elles recrutent des candidats qui ont été formés dans des universités anglophones. La didactique est une discipline qui a été développée dans le monde francophone et plusieurs de ses concepts n'ont pas d'équivalent dans le monde anglophone.

De plus, les spécialistes des autres domaines des sciences de l'éducation et les divers acteurs du milieu de l'éducation ne comprennent pas toujours les orientations et les objets d'étude qui rendent la didactique incontournable. Il n'est pas rare, par exemple, d'entendre des acteurs du milieu scolaire faire des affirmations du genre « un bon enseignant peut enseigner n'importe quelle matière » ou " la didactique d'une matière scolaire, c'est l'étude des 'trucs et astuces' pour mieux enseigner cette matière ». Ils préfèrent donc, par exemple, adhérer au nouveau « dogme» des «données probantes ». Mais ces données probantes portent sur l'efficacité de pratiques tellement générales (exemples: exposés par l'enseignant, travail en équipe, rétroaction) qu'elles ont peu de retombées dans les classes 
et sont très peu utiles pour aider les élèves à surmonter les obstacles propres à chacune des matières scolaires.

Enfin, pour réduire les couts, certaines universités, même parmi les plus grandes, offrent des programmes de deuxième et de troisième cycles, en didactique, qui ne comportent aucun cours obligatoire portant sur les théories et les concepts de la didactique ou sur les méthodes de recherche en didactique. Sans doute parce qu'elles ne reconnaissent pas les particularités de la didactique et de la recherche dans ce domaine, elles postulent, à tort, que les étudiants peuvent se contenter de cours obligatoires portant sur les concepts et les types de recherche communs à toutes les disciplines des sciences de l'éducation. Alors adieu contrat didactique, transposition didactique, niveaux de formulation, modèles du changement conceptuel, ingénierie didactique, conscience disciplinaire et autres notions fondamentales de la didactique dans la formation des étudiants des cycles supérieurs (sauf si le directeur ou la directrice de la recherche prend en charge toute cette formation).

\section{La revalorisation de la didactique}

En raison de ces menaces constantes, les didacticiens peinent à contribuer autant qu'ils le souhaiteraient à l'amélioration de l'enseignement et de l'apprentissage des diverses disciplines scolaires. Cela explique peut-être en partie que la grande majorité des élèves, et même ceux qui décident de faire des études postsecondaires, abandonnent rapidement les matières scolaires (exemples : mathématiques, physique, biologie) dans lesquelles les savoirs contemporains sont les plus éloignés des connaissances primordiales mentionnées au début de l'article. Au Québec, par exemple, près de 70\% des élèves ne s'inscrivent pas aux cours de mathématiques et de sciences les plus avancés de la dernière année du secondaire. Par comparaison avec le degré d'avancement de ces disciplines dans la communauté scientifique, ces cours n'en sont pourtant que des bases très rudimentaires.

La conviction de plusieurs didacticiens est d'ailleurs qu'il y a beaucoup trop de recherches et de publications, en sciences de l'éducation, qui portent sur des thèmes qui n'ont rien à voir avec l'essentiel, qui devrait être la meilleure façon d'enseigner et d'apprendre chacune des matières scolaires. Même dans les facultés ou départements de sciences de l'éducation, certains professeurs et administrateurs s'étonnent parfois que des équipes de recherche en didactique puissent consacrer des années à étudier l'enseignement et l'apprentissage de notions en apparence aussi minuscules que l'emploi des prépositions, la division des fractions, les phases de la Lune, la théorie cellulaire, le modèle particulaire, le document historique ou la perspective. C'est mal comprendre à quel point tous les concepts enseignés dans une école du $21^{\mathrm{e}}$ siècle se situent à des années-lumière des connaissances primordiales de l'Homo sapiens et exigent d'énormes sauts conceptuels de la part des élèves. 
On peut donc affirmer qu'il serait essentiel de revaloriser la didactique, de mieux la protéger de tout ce qui peut la menacer, et de lui accorder une place beaucoup plus grande dans les programmes d'études des facultés et départements de sciences de l'éducation, dans les domaines de spécialisation du corps professoral ainsi que dans les axes de recherche privilégiés par les organismes subventionnaires.

En terminant, nous espérons que cet article aura permis au lecteur de se familiariser avec les connaissances primordiales des êtres humains, de comprendre comment la didactique peut être définie, en extension partielle, au moyen des théories et des concepts qui contribuent à tenir compte de ces connaissances primordiales, et de se sensibiliser à certaines menaces qui pèsent sur la didactique et en constituent également des contreexemples.

Note: La section de cet article qui porte sur les théories et concepts de la didactique est adaptée du deuxième chapitre de l'ouvrage "Réaliser une recherche en didactique » écrit par l'auteur. (Voir la référence complète ci-dessous.)

\section{Remerciements}

L'auteur tient à remercier ses collègues Marie-Claude Boivin et Annette BraconneMichoux pour leurs commentaires et suggestions.

\section{Références}

Artigue, M. (1996). Ingénierie didactique, Dans J. Brun, Didactique des mathématiques, Paris : Éditions Delachaux et Niestlé. 243-274.

Astolfi, J.-P. (1997). L'erreur, un outil pour enseigner. ESF Éditeur.

Astolfi, J.-P., Darot, É., Ginsburger-Vogel, Y. et Toussaint, J. (2008). Mots-clés de la didactique des sciences. Éditions De Boeck.

Bachelard, G. (1934/1993). La formation de l'esprit scientifique. Éditions Vrin.

Bardin, L. (2003). L'analyse de contenu. Presses Universitaires de France.

Boilevin, J.-M. (2013). Rénovation de l'enseignement des sciences physiques et formation des enseignants. Éditions De Boeck.

Brousseau, G. (1983). Les obstacles épistémologiques et les problèmes en mathématiques. Recherches en Didactique des Mathématiques. 4 (2), 165-198.

Brousseau, G. (1986). Fondements et méthodes de la didactique des mathématiques. Éditions La Pensée Sauvage.

Brousseau, G. (1998). Théorie des situations didactiques. Éditions La Pensée sauvage. 
Chevallard, Y. (1985/1991). La transposition didactique: Du savoir savant au savoir enseigné. Éditions La Pensée Sauvage.

Comte-Sponville, A. (2013). Dictionnaire philosophique. Presses Universitaires de France. Cornu L. et Vergnioux A. (1992). La didactique en questions. Hachette Éducation.

De Vecchi, G. (2007). Enseigner par situations-problèmes. Éditions Delagrave.

Develay, M. (1991). Discipline et « matrice disciplinaire », Cahiers pédagogiques, $\mathrm{n}^{\circ} 298$, Les contenus d'enseignement, Paris, CRAP, 25-27.

DiSessa, A. (1993). Toward an epistemology of physics, Cognition and Instruction, 10 (2 et 3), 105-225.

Fédier, F. (2006). L'art en liberté. Éditions Pocket.

Gauthier, C. (1991). L'insoutenable légèreté de la pédagogie, Revue des sciences de l'éducation, 17(2), 283-295.

Kundera, M. (2000). Les testaments trahis. Folio.

Migne J. (1969). Les obstacles épistémologiques et la formation des concepts, Éducation permanente, Vol. 2. 39-66.

Ohlsson, S. (2009). Resubsumption : A possible mechanism for conceptual change and belief revision. Educational Psychologist, 44 (1), 20-40.

Pinker, S. (2002). The Blank Slate. Viking Penguin Books.

Posner, G. J., Strike, K. A., Hewson, P. W. et Gertzog, W. A. (1982). Accomodation of a scientific conception : Toward a theory of conceptual change, Science Education, 66 (2), 211-227.

Potvin, P., Sauriol, É., et Riopel, M. (2015). Experimental evidence of the superiority of the prevalence model of conceptual change over the classical models and repetition. Journal of Research in Science Teaching, 52 (8), 1082-1108.

Potvin, P. (2018). Faire apprendre les sciences et la technologie à l'école. Épistémologie, didactique, sciences cognitives et neurosciences au service de l'enseignant. Les Presses de l'Université Laval.

Reuter, Y. (dir.) (2013). Dictionnaire des concepts fondamentaux des didactiques, $3^{e}$ édition. Éditions De Boeck.

Robert, A. et Butlen, D. (2012). Les recherches en didactique des disciplines : une nécessité actuelle? Dans M.-L. Elalouf, A. Robert, A. Belhadjin et M.-F. Bishop, Les didactiques en question(s) : État des lieux et perspectives pour la recherche et la formation (p. 21-32). Éditions De Boeck.

Thouin, M. (2014). Réaliser une recherche en didactique. MultiMondes.

Thouin, M. (2017). Enseigner les sciences et les technologies au préscolaire et au primaire, $3^{e}$ édition. MultiMondes.

Vergnaud, G. (1990). La théorie des champs conceptuels, Recherche en didactique des mathématiques, 10 (23), 133-170. 
Vosniadou, S., et Brewer, W. F. (1992). Mental models of the earth: A study of conceptual change in childhood. Cognitive Psychology, 24 (4), 535-585.

Vygotsky, L. S. (1934/1997). Pensée et langage. La Dispute.

Vygotsky, L. S. (1931/1985). La méthode instrumentale en pédagogie, Dans B. Schneuwly et J.-P. Bronckart (dir.), Vygotsky aujourd'hui (p. 39-47). Delachaux-Niestlé. 